\title{
INFORMATION FUSION ARCHITECTURES FOR NEXT GENERATION AVIONICS SYSTEMS
}

\author{
Victor Clark \\ System Integration Branch \\ Wright Laboratory, WL/AAAS, Bldg. 620, 2185 Avionics Circle \\ WPAFB, OH 45433-7301
}

\begin{abstract}
In 1994, the Avionics Directorate of Wright Laboratory formed an Information Fusion Working Group (IFWG) to examine current fusion work, and identify future directions that the directorate should pursue in the fusion area. The first result of the IFWG was the formation of an Avionics Directorate Information Fusion (IF) Integrated Product Team (IPT) to help coordinate the fusion efforts in the directorate. In addition, the IFWG made four specific recommendations to enhance the avionics fusion research area. These four recommendations are as follows: 1) Develop an open modular avionics information fusion architecture, 2) Develop fusion interface standards; 3) Develop metrics to evaluate fusion algorithms, and 4) Develop an open fusion testbed. This paper will focus on the development of an open avionics information fusion architecture. It will describe some of the issues associated with the development of an avionics fusion architecture and will culminate into a description of our current avionics information fusion architecture design. This fusion architecture design is based on the four level fusion model that was defined by the Joint Director of Laboratories (JDL) Data Fusion Sub-panel. It is our intent to develop an open fusion architecture that will allow system designers the capability to re-use existing fusion algorithms. This will be accomplished through the use of modular object-oriented software engineering techniques. Where possible, emerging industry standards, such as the Common Object Request Broker Architecture, the database standards from the Object Database Management Group, and others will be used to facilitate the broadest community acceptance. The IF IPT architecture design has been an evolving process in which the current architecture design has been presented to members of the fusion community and their comments have been incorporated into the overall design. The IF IPT hopes to continue this process through the implementation stage to facilitate fusion community support.
\end{abstract}

\subsection{INTRODUCTION}

In early 1994 the Avionics Directorate of Wright Laboratory formed an Information Fusion Team for
Avionics (IFTA) to coordinate fusion efforts and to determine future fusion research directions in the organization. In order to determine the current state of fusion research, the team surveyed a large number of existing fusion efforts to determine the different levels of fusion that were taking place. In addition, the IFTA also formed an Information Fusion Working Group (IFWG) made up of senior level fusion researchers to aid in the analysis of the fusion surveys and to help formulate future fusion research directions. The IFTA/ IFWG made four specific recommendations to enhance the "state of the art" in the avionics information fusion research area. They are as follows:

1) Develop an open avionics fusion architecture.

2) Develop open interface standards for fusion systems.

3) Develop open metrics to evaluate fusion algorithms

4) Develop an open fusion testbed.

In order to accomplish these objectives and coordinate fusion research in the Avionics Directorate, an Information Fusion Integrated Product Team (IFIPT) was formed. This paper describes the design of an avionics information fusion architecture. This design was developed by the Information Fusion Integrated Product Team. The IFIPT has taken the lead on this design effort to insure an independent open architecture that is not tied to one specific contractor. Hopefully this will lead to broader community support because individual contractors will not have to endorse their competitors concepts. It is not the intent of this effort to inflict rigid unyielding standards, but rather a set of engineering design guidelines that will enhance the fusion community and provide a starting point for some commonality between the different fusion efforts. These "standards" will be enhanced and modified over time as the community becomes smarter in this area. In addition, it is not the intent of this effort to dictate specific fusion algorithms, but rather define broad and flexible container classes and interfaces that will allow algorithm re-use.

\subsection{DESIGN ISSUES}


In order to facilitate fusion algorithm reusability, modular software engineering techniques must be incorporated into the overall fusion architecture design. Clearly, which ever form our final architecture takes, it is going to be based on a modular, object-oriented, message based design. This design push is coming from the need for increased processing demand, parallel processing and the emerging of standards such as CORBA, ODMG, and DIS. Even computer languages such as $\mathrm{C}++$, Ada 95, and Object Pascal are organized around an object oriented, message passing paradigm. With this in mind, a set of guiding design principles were established to insure an open flexible architecture. The guiding principles that we used to lay out this architecture are as follows:

\section{Object-oriented modular design.}

2. Levels of abstraction - partitioning the processes in such a fashion that the information is abstracted as it goes from the lower to upper objects.

3. Balance of Breath vs. Depth - objects should be defined in such a way as to minimize possible bottlenecks i.e. where there is a lot of depth of knowledge, minimize breath; higher level objects have more breath less depth.

4. Levels of Hierarchy - This architecture should be able to accommodate alternative design approaches. Therefore metrics and interfaces should be estabiished at each level so that system designers with alternative techniques could replace a function object and still interact with the rest of the architecture.
In addition to the object oriented design, the Levels of abstraction, and Balance of Breath vs. Depth design goals were developed to eliminate possible bottlenecks in the system. One of the difficulties associated with earlier fusion efforts is that system level control was too centralized through the use of all encompassing mission managers or centralized blackboard schemes. This design attempts to partition the control and processor load throughout the whole system. In the IFIPT fusion architecture design, the modules with the most in-depth algorithmic processing have less system level breath. Conversely, the upper level modules which have more symbolic processing tend to encompass more breath, but they rely on the lower level modules to provide the depth of knowledge. Lastly, this architecture design is based on a hierarchical scheme. This hierarchical scheme is based loosely on the object-oriented concept of a child class. Under this design, more specific children classes can be removed and replaced with legacy code as long as the system developer adheres to the parent container class and interfaces. This will allow the system designer to grow into the modular layout of the open architecture without scrapping their current code. In addition, it will also provide a way for the architecture to expand as the technology grows.

\subsection{ARCHITECTURE DESIGN}

Based on the design principles stated above, the IFIPT has laid out a fusion design based on the JDL's Data Fusion sub-panel architecture (Waltz and Llinas p. 15). This architecture, figure 1, encompasses all four fusion leveis. In conjunction with the design principles, the objects or container classes at the bottom tend to be more algorithmic in nature. In addition, these objects focus in on more

\section{Avionics Information Fusion Architecture}

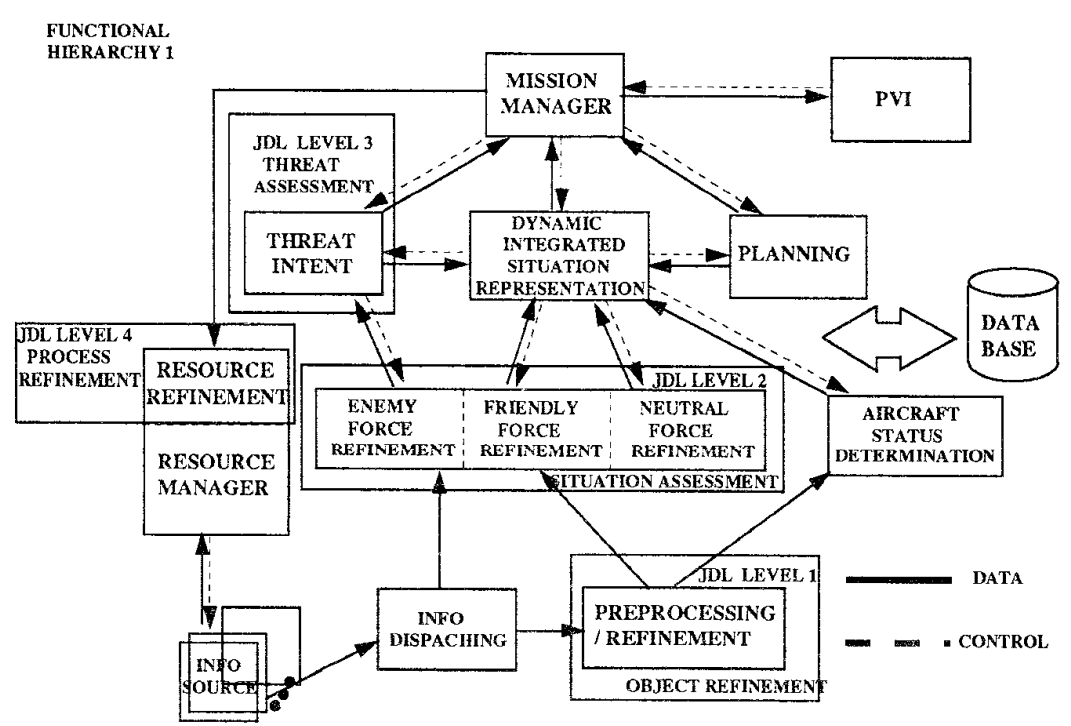

Figure 1 
detailed information about the sensors. As the information flows upward in the system, it tends to take on a more symbolic nature which is focused less on the specific details and more on higher level fusion goals.

With this in mind, figure 1 represents a functional objectoriented message passing layout of our top level system. This top level is defined as the "Functional Hierarchy 1 " level. Each of the boxes that make up the top level, can be thought of as an aggregate collection of processes. Inside each one of the aggregated object there are sub-objects or child classes. Composing each sub-object are either further sub-objects or methods which hold the individual fusion algorithms. In addition to the upper level objects, the top level diagram also defines the high level information flow in the system. In an attempt to abstract the data as it flows from the lower levels to the upper level, the data tends to flow upward and the control signals tend to flow lower. In this fashion, the data at the lower levels start out with the sensor data and abstracts to higher levels of reasoning as it proceeds to the mission manager and to the aircrew.

At the lowest level of our architecture design, there is an input for information sources including both on-board and off-board data. This sensor data goes into an information dispatching object. The purpose of the information dispatching object is to route the incoming on-board and off-board data to the appropriate fusion level. In the case of on-board data, the sensor data would be sent to the Preprocessing/ Refinement object to go through level 1 processing. However, in the case of off-board intel data, the incoming data might have already gone through some level 1 processing and be ready to be incorporated into level 2 , situation assessment. The information dispatching box is responsible for determining which fusion level the data is arriving at and insuring the data gets sent to the appropriate level.

Avionics Information Fusion Architecture

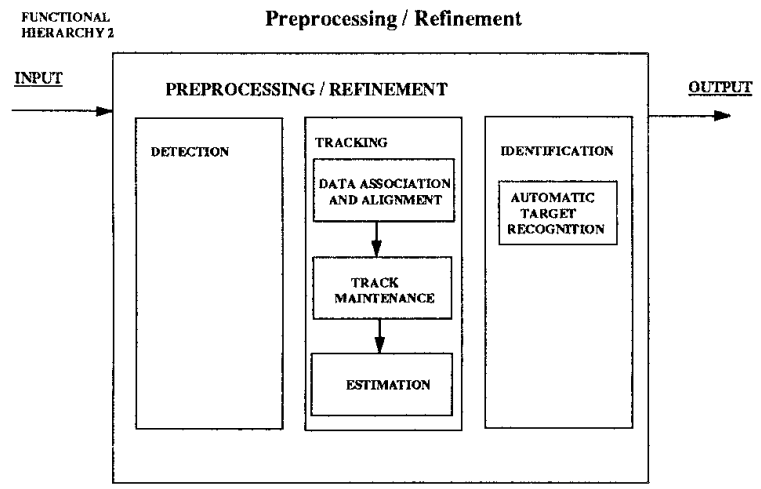

Figure 2
After the Information Dispatching object, the next object of interest is the Preprocessing / Refinement Object. The purpose of the Preprocessing/ Refinement Object is to process sensor data through the detection, tracking and identification stages to identify individual objects and their kinematic characteristics. The output of this level would be individual objects such as a single aircraft, tank or other object. The Preprocessing/ Refinement object is broken into three sub-objects or child classes. They are the Detection, Tracking and Identification Objects. These three objects define the "Functional Hierarchy 2" level for the Preprocessing/ Refinement object. The Tracking and Identification Objects are further broken down into the Data Association, Track Maintenance, Estimation and ATR subobjects. These four sub-objects define the "Functional Hierarchy 3" level for the Preprocessing/ Refinement Object. Under the Levels of Hierarchy design concept, if the system designer's fusion algorithms do not fit in the lower functional hierarchical level, they are free to remove the child container class, and implement their algorithms in the parent container class. For instance, a fusion designer could easily implement a Nearest Neighbor or Joint Probabilistic Data Association Algorithm in the Data Association and Alignment sub-object. If however, a new tracking algorithm was developed that did not fit into the Data Association, Track Maintenance, and Estimation subobjects, these sub-objects could be removed and the new tracking algorithm could be implemented at the Tracking object level. The only constraints on the fusion designer would be to adhere to the interfaces and container classes of the parent object. 'The same concept also applies to the higher level objects. If a new Level 1, Preprocessing/ Refinement algorithm was developed, the system designer could remove the Detection, Tracking, and Identification objects, and implement the algorithm at the Preprocessing / Refinement container class. In this manor, maximum flexibility can be maintained, and portions of legacy code can be utilized.

Avionics Information Fusion Architecture

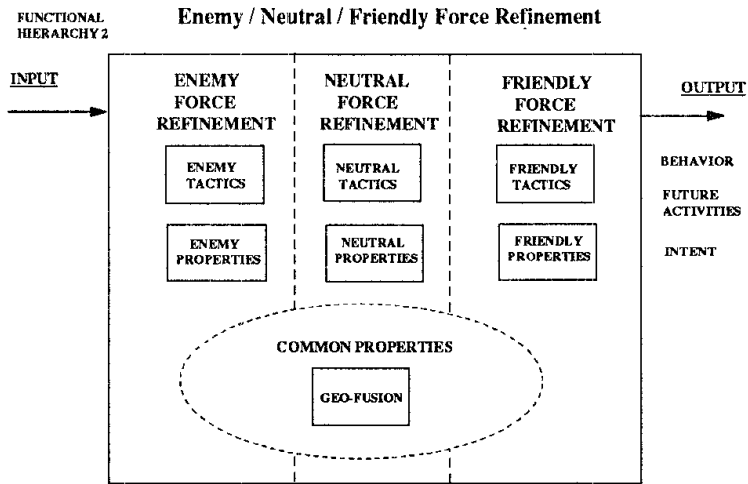

Figure 3 
Once the individual objects are identified, they are passed to the level 2, Situation Assessment object, figure 3, to be grouped or clustered together. The output of the Situation Assessment object would be a grouping of objects such as an aircraft wing or a tank column. The Situation Assessment object is divided into three major components: Enemy Force Refinement, Friendly Force Refinement, and Neutral Force Refinement. In addition there is a common object that spans all three areas called the geo-fusion object. The geo-fusion object is responsible for reasoning about the geographical properties of the individual objects (i.e. tanks don't drive in the middle of lakes, etc.). In addition, each major component has a tactics object which reasons about the corresponding friendly or enemy tactics. Similarly, the properties object reasons about the corresponding properties and kinematics of the composite elements. In addition to the Enemy and Friendly Force Refinement Objects, there is also a Neutral Force Refinement Object. The Neutral category is more of a "catch all" area to cluster objects which do not easily fit into one of the other categories. For instance, just because the Level 1, Preprocessing object identifies an aircraft as an F-16, it can not be assumed that this is a friendly aircraft. For lack of a better term, the Neutral Force Refinement object was added to address unique concerns. Additionally, each one of these individual sub-objects or container classes that make up the Situation Assessment object are defined at the "Functional Hierarchy 2" level. There is no "Functional Hierarchy 3" level definition for this module.

\section{Avionics Information Fusion Architecture}

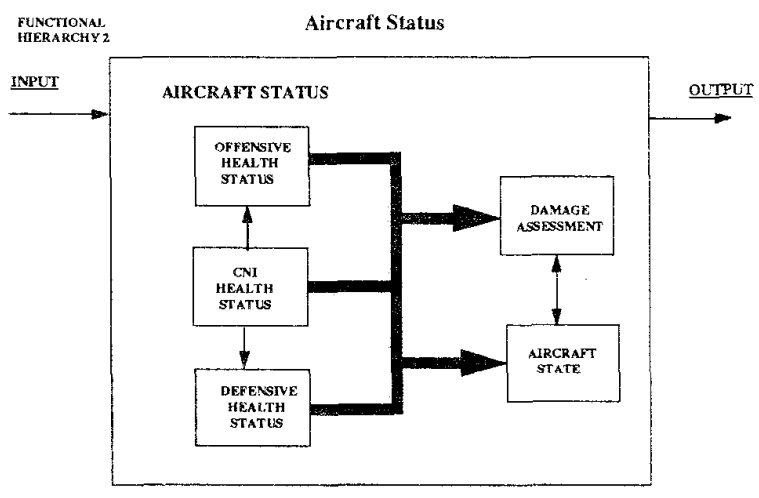

Figure 4

Next to the Situation Assessment object is the Aircraft Status object, Figure 4. Though this function is not part of the original JDL fusion model, it was added to this architecture to provide status information about the onboard sensors to the rest of the system. As seen in figure 4, Aircraft Status is broken into offensive sensors, defensive sensors, and the CNI sensors. The health of these different sensors are fed into an Aircraft State object which collects this information and provides a unified sensor status. In addition, there is Damage Assessment object which takes into account if a particular sensor is damaged or being jammed. This object is a mini planning module which attempts to overcome degraded sensor performance by utilizing alternative sensors. For instance, if one of the four GPS satellites that are being used by the navigation system gets jammed, the Damage Assessment object might suggest the use a radar altimeter to replace the jammed satellite.

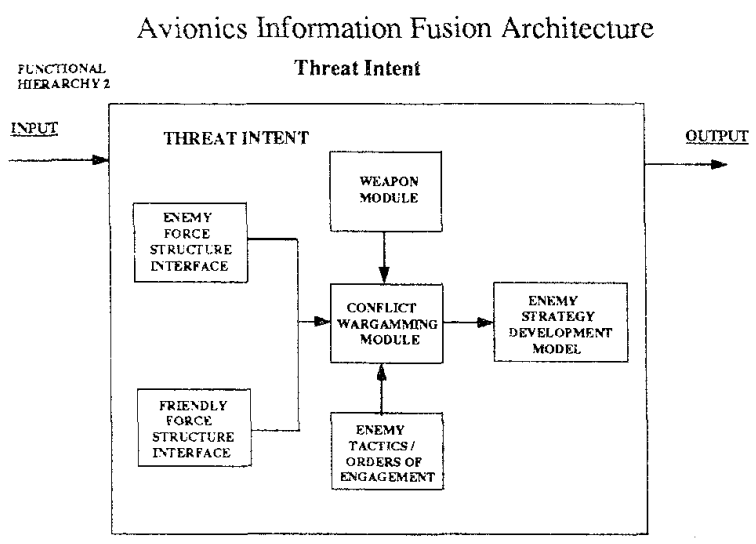

Figure 5

After the fusion information is processed through the Situation Assessment object, the composite information is sent to the Threat Intent object show in figure 5. The Threat Intent object attempts to collect the composite information generated by level 2 fusion and reason about the lethality on the composite objects. At the heart of the Threat Intent module is the Conflict Wargaming Module. While it is not the intent of this paper to dictate possible implementation algorithms, it is envisioned that a neural network with adversarial roles might be used to model the interaction of the friendly and enemy forces. The input to the wargaming module would come directly from the Enemy Force Structure Interface and the Friendly Force Structure Interface Objects. The purpose of these two interface objects is take the outputs from the level 2 , Situation Assessment object and manipulate it in such a way that they can be used by the Conflict Wargaming Module to predict enemy actions. In addition to the two interface objects, there is also a Weapons Module and an Enemy Tactics/ Order of Engagement Module. The Weapons Module is responsible for modeling the weapons interaction between the two opponents. This information is fed directly into the Wargaming object. Also aiding the Wargaming module is the Enemy Tactics/ Order of Engagement module. This module is envisioned as a knowledge based module, which holds heuristics information about the enerny's tactics and any constraints placed on the conflict due to their engagement tactics. The output of the Conflict Wargaming Module are fed into the Enemy Strategy Development Model object. This object is responsible for 
taking adversarial data that is being generated by the Conflict Wargaming Module, and using this information to predict the future intent of the enemy forces. The subobjects that make up the Threat Intent Object are defined to the "Functional Hierarchy 2" level. The output of the Threat Intent object feeds directly into the one of the major components of the fusion architecture, the Dynamic Integrated Situation Representation (DISR) object. However, before describing the DISR object, it is important to understand the inputs generated by the Planning object.

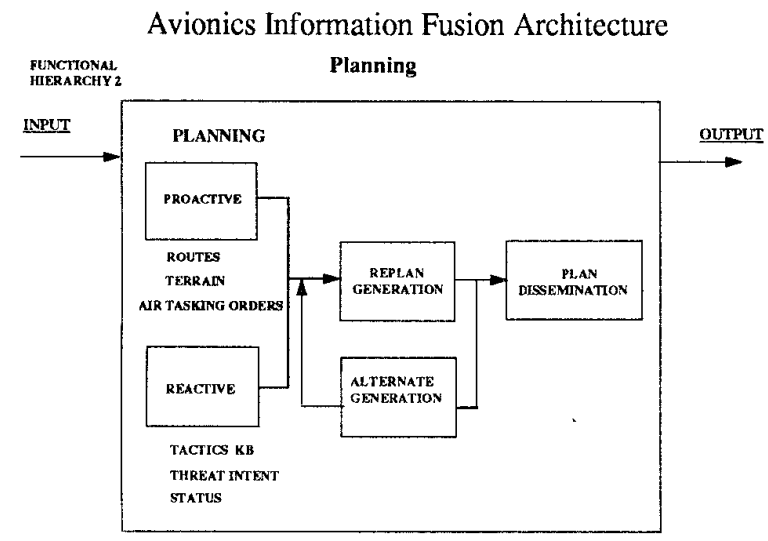

Figure 6

The Planning object, figure 6 , is responsible for analyzing the level 1,2, and 3 fusion data and making recommendations to the aircrew to improve their situational awareness. Although, this module was not part of the original JDL fusion architecture, it was felt that the planning module would be beneficial to provide a more offensive flavor to the avionics fusion architecture. The Planning module provides two major types of planning, Proactive and Reactive. The Proactive sub-object is responsible for providing the necessary expertise for the mission planning type functions. These functions would include route planning, terrain following, tasking orders execution, and avoiding pop up threats. The Reactive sub-object would be responsible for providing the expertise for more of the tactics type planning functions. These functions would include more tactics and treat interaction. This expertise would be fed into a bank of planning objects that would be responsible for generating a set of planning scripts and alternate planning scripts. In figure 6 , this bank of planning objects is denoted by the Re-plan Generation object and the Alternate Generation object; however, there is no need to limit the system to two planning modules, if the required processing resources are available. The individual sub-plans are fed into the Plan Dissemination Object in order to come up with a cohesive plan. In this fashion, the planning object would suggest to the pilot the best mission route and help the pilot determine which enemy target is the most lethal. It is not the intent of this design effort to develop a large
Pilot's Associate type system. However, with the Levels of Hierarchy design principles, more robust planning and EW functions could be added to the Planning object. The Planning object interacts directly with the Mission Manager object and the Dynamic Integrated Situation Representation (DISR) object.

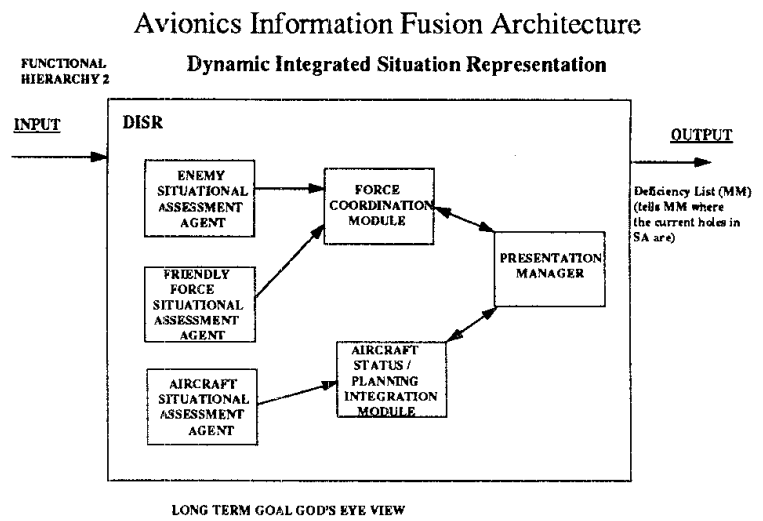

Figure 7

The DISR object, figure 7, is one of the key components of the avionics fusion architecture. The DISR receives input from the Level 2, Situation Assessment object, the Level 3, Threat Intent object, the Aircraft Status Determination object, and the Planning object. As might be concluded from this level of connectivity between the objects, the DISR is responsible for coordinating all the information from the different levels and presenting this information in the clearest possible form to the aircrew. The other major function of the DISR is to identify any deficiencies in the current aircraft situational awareness, and to pass this information through the Mission Manager to the Resource Manager. These functions are accomplished by partitioning the DISR into two parts. The upper part, which is made up of the Enemy Situational Assessment Agent, the Friendly Force Situation Assessment Agent, and the Force Coordination Module are responsible for pulling together the level 2 and 3 information to get a complete picture of where the friendly and enemy force structures are located and their intent. The lower part of the DISR is made up of the Aircraft Situation Assessment Agent and the Aircraft Status/ Planning Integration Module. These two objects are responsible for coordinating the aircraft sensor health and the proactive/ reactive planning of the fusion system. Both the upper and lower parts feed into the DISR's Presentation Manager. The Presentation Manager object pulls together these two parts and sends this coordinated information to the aircrew. In addition, because of the coordinated view, the Presentation Manager object would also be in a position to identify any deficiencies in the fusion system. Although the DISR was not explicitly defined in the original four level fusion model, its functionality has been elevated to a higher level due to the importance and complexitics 
associated with its functions. The outputs of the DISR are passed through the Mission Management to either the Pilot Vehicle Interface (PVI) object or the Resource Management object depending on the content of the message.

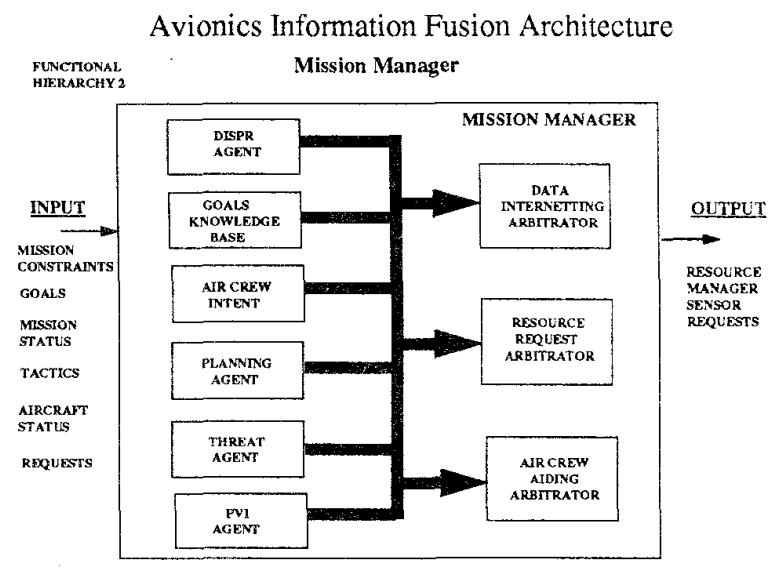

Figure 8

At the top of the avionics fusion architecture design is the Mission Manager object, figure 8. Consistent with the second and third design guidelines, Levels of abstraction and Balance of Breath vs. Depth, the Mission Manager focuses on the high level goals of the fusion system. In an attempt to eliminate any possible bottlenecks in the system, the Mission Manager relies on the lower level objects to provide the depth in the system. The Mission Manager's primary purpose in the system is to serve as a master arbitrator to protect the two most critical resources, the aircrew and the sensors. In order to accomplish these goals, the Mission Manager has intelligent agents which interact with the DISR object (through the DISR Agent), the Threat Intent Object ( through the Threat Agent), and the Planning object (through the Planning Agent). In addition, it contains an intelligent Goals Knowledge Base object which would contain the high level goals of the mission, any preflight planning information, and any aircrew preferences. Likewise, it contains an Aircrew Intent object to attempt to determine the level of aircrew workload in order to insure that the right type of information is being sent at the right time to the pilot. All of the above information flows into the two arbitrator objects, the Resource Request Arbitrator object and the Air Crew Aiding Arbitrator. The Air Crew Aiding Arbitrator works with the DISR to insure that the right type of fusion information is being sent to the aircrew. It also uses inputs from the Air Crew intent object to insure that the right type of data is arriving at the proper mission phase. The Resource Request Arbitrator object insures that the sensors usage adheres to, and helps to advance the upper level goals of the mission. In addition, the Resource Request Arbitrator works closely with the Data Internetting Arbitrator object. The Internetting Arbitrator is responsible for managing information request between platforms. It is envisioned that multiple aircraft will be able to share sensor data. This could be used to accomplish a cooperative hunter shooter type mission, pass data between an aircraft and wingman, or even pass data between a fighter and an AWACS. In either case, whenever data is requested from an outside source, one of the two most critical resources, the aircrew or the sensors are being requested. When this occurs, the Internetting Arbitrator would work with the Resource Request Arbitrator, the Aircrew Aiding Arbitrator, and the Goals Knowledge Base to determine whether to grant the request. Finally, the output of the Mission Manager either goes to the PVI to be send to the aircrew or to the Resource Manager object to schedule a sensor update. In either case the Mission Manager deals with very abstracted, highly symbolic data which should not result in a bottleneck in the system.

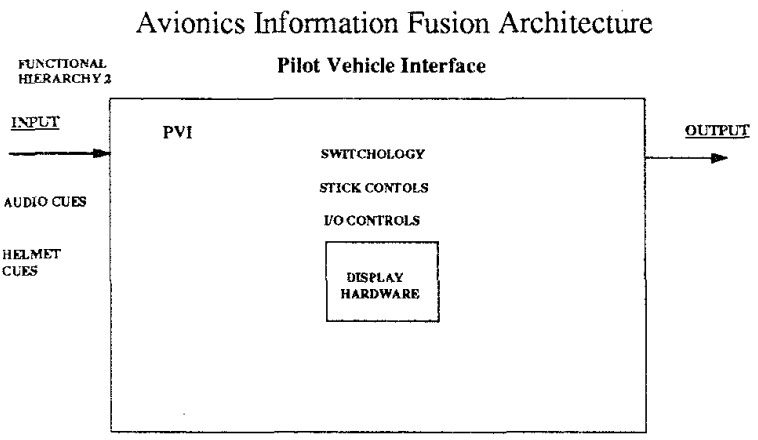

Figure 9

In an embedded environment, the Mission Manager of the Information Fusion system will send data directly to an overall mission manager of the aircraft. Therefore, this architecture design does not really address the concept of aircraft displays (Figure 9) beyond the notion of hardware displays. For the early phases of the architecture development, it will be necessary to implement these graphical displays in a laboratory environment. With this in mind it is time to examine the last object that makes up the fusion architecture, the Resource Manager object.

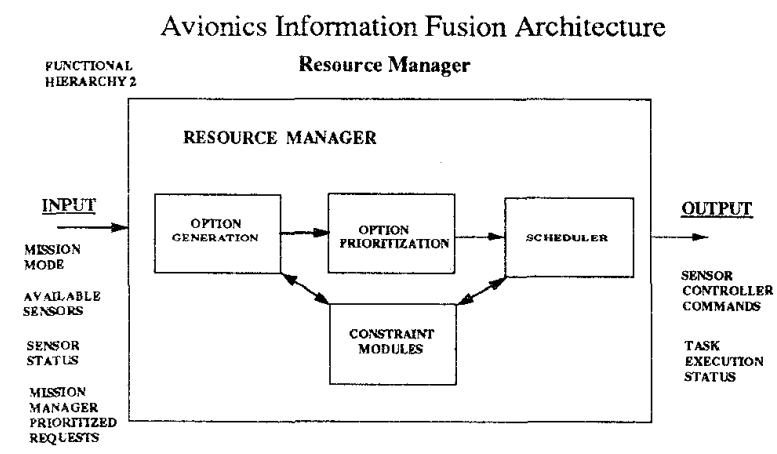

Figure 10 
The Resource Manager object, figure 10, falls under JDL's level 4 fusion. The Resource Manager receives requests from the Mission Manager and sends commands to the sensors. Although the Resource Manager performs level 4 fusion, it is placed under the Mission Manager in the Function Hierarchy level 1 layout, figure 1, because it deals directly with the sensors and uses lower level, algorithmic data. The Resource Manager is broken down into three major components, the Option Generation object, the Option Prioritization object, and the Scheduler object. These three objects operate in a very similar fashion to the Planning Module. However, instead of focusing on the mission and tactics planning, the Resource Manager generates segment scripts to carry out portions of the sensor queuing and update cycles. The Option Generation object is the module that generates these individual sub scripts based on the sensor requests received from the Mission Manager. Once these scripts are generated, they are pasted to the Option Prioritization object to prioritize the ranking of the individual scripts. Once this is accomplished, the Scheduling option takes the prioritized set of tasks and schedules them for execution by the sensors. In addition to the three objects described above, the Resource Manager also has a Constraint Manager object to insure that the generated plans do not violate any constraints imposed on them by the rest of the system.

\subsection{IMPLEMENTATION STRATEGIES}

In order to implement an object oriented avionics architecture, there is a whole spectrum of implementation methodologies that could be used. Towards the language side of the implementation scale, an object oriented language such as $\mathrm{C}++$, Ada 95 or Object Pascal could be used to implement an open fusion system.

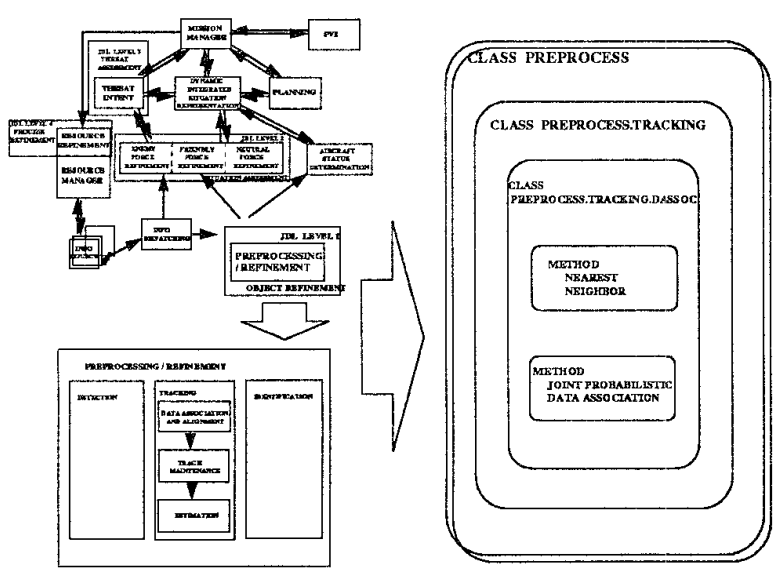

Figure 11

For example, in figure 11, a child class structure is used to implement the Data Association sub-class, which is contained in the Tracking child class, which is part of the
Preprocessing base class. In this manner, if the system designer is developing a conventional Nearest Neighbor algorithm, it would fit nicely in the Preprocessing.Tracking.Dassoc class. However, as new tracking algorithms are developed, the child Dassoc child class could be removed and the algorithm could be implemented in the Preprocessing.Tracking class. Likewise, the Tracking child class could also be removed, and the algorithms could be implemented directly in the Preprocessing base class. Finally, on the opposite spectrum of implementation strategies, a distributed blackboard or communicating expert object mechanism could be used to implement the system.

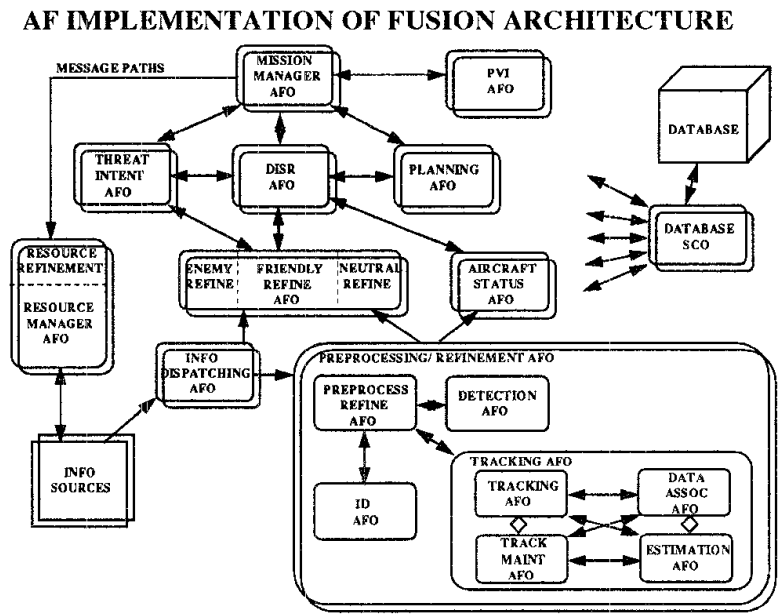

Figure 12

As seen in figure 12, each upper level object in the system is an aggregated collection of sub-objects. A closer examination of the Preprocessing object reveals that it is made up of ID, Tracking, and Detection objects. Likewise, these sub-objects are in themselves an aggregated collection of objects. In this fashion, if it is not possible to implement an algorithm in the Data Association sub-object, the individual sub-objects at that level could be removed, and the parent objects could be used to implement the fusion algorithms. This implementation strategy is made possible through the design principle of "Levels of Hierarchy" and the use of good object-oriented software engineering techniques. Along the lines of good software engineering techniques is the need for algorithm reusability. Whichever design approach is selected, it must insure that the algorithms can be kept an a flexible, modular format that can be easily reused in future projects. One popular format that is being used by simulation tools such as Math Work's Simulink or Khoros is to use graphical hierarchical storage techniques. These techniques give users the ability to define the algorithms and interfaces in a modular, reusable graphical format. Once these objects are defined, a user can 


\section{HYBRID SIMULATION ENVIRONMENT}

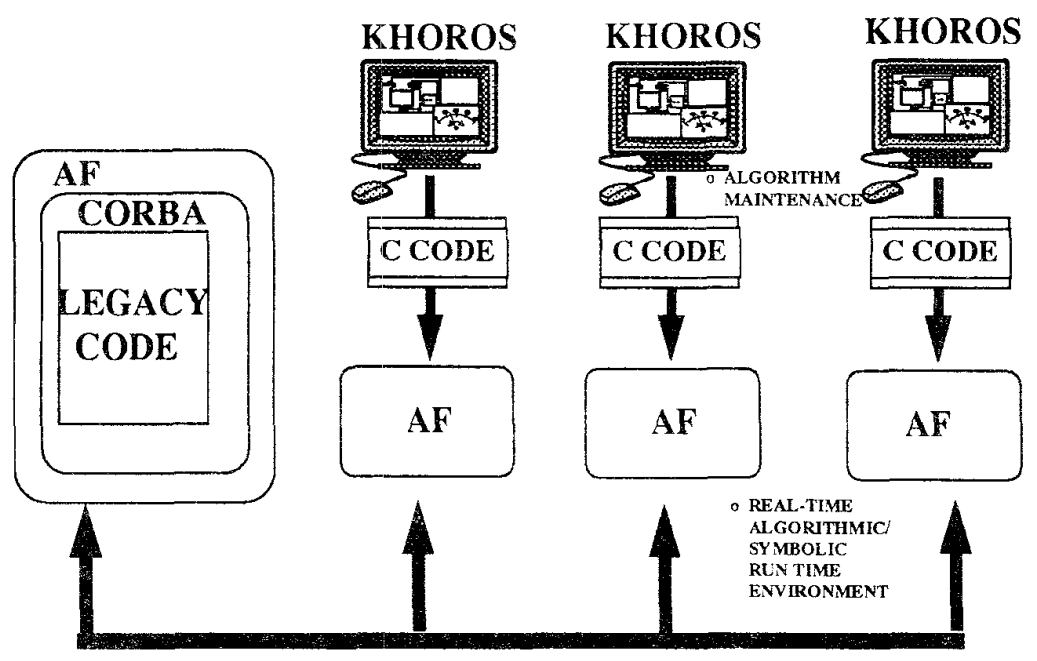

Figure 13

graphically connect the pieces together to build up different sub-graphs or entire fusion systems. One possible way of pulling together these components is shown in figure 13. Note however, that this is only a conceptual approach which is being used to illustrate reuse concepts. Under this conceptual approach, the algorithm developer could maintain the fusion algorithms in very modular, re-useable graphical format. Once the appropriate fusion algorithms have been laid out, $C$ code can be generated and feed into a real-time distributed architecture to execute. The fusion designer could use CORBA or some other industry emerging standard to wrap around any legacy code they may be using.

\subsection{CONCLUSION}

The Avionics Directorate IFIPT is attempting to develop an open avionics fusion architecture. This paper describes an object oriented modular design for this architecture based on the JDL Data Fusion Sub-Panel's four level fusion model. This design is based on four guiding principles. They are as follows:

1. Object-oriented modular design.

2. Levels of abstraction.

3. Balance of Breath vs. Depth.

4. Levels of Hierarchy.

These guidelines were established to insure maximum flexibility and reusability in the system. By building on the JDL's Data Fusion efforts, a common set of fusion containers and interfaces can be developed to utilize and reuse existing fusion algorithms. This reuse will result in significant time and resource savings. It is not the intent of this effort to inflict rigid standards on the fusion community, but rather to leverage existing commercial, government and university practices whenever possible.

\subsection{BIBLIOGRAPHY}

Bowman et al., "Information Fusion Technology Plan Executive Summary", Wright Patterson AFB, OH, 1995.

Green, Peter, "Shell for Converting Knowledge Representations in Ada with Easy Maintainability", Wright Lab Tech Report WL-TR-94-1117, Wright Patterson AFB, $\mathrm{OH}, 1994$.

Hall, David L., Mathematical Techniques in Mutlisensor Data Fusion Artech House, Boston, MA, 1992.

Khoral Research, KHOROS Foundation Services, Khoral Research, Inc, Albuquerque, NM, 1994.

Waltz, E. , Llinas J, Multisensor Data Fusion, Artech House, Boston, MA, 1990. 